\title{
Supermassive Black Holes
}

\author{
Kenneth Dalton \\ Independent Researcher, Bang Saphan, Thailand \\ Email:kxdalton@yahoo.com
}

How to cite this paper: Dalton, K. (2019) Supermassive Black Holes. Journal of High Energy Physics, Gravitation and Cosmology, 5, 984-988.

https://doi.org/10.4236/jhepgc.2019.53052

Received: October 9, 2018

Accepted: July 28, 2019

Published: July 31, 2019

Copyright $\odot 2019$ by author(s) and Scientific Research Publishing Inc. This work is licensed under the Creative Commons Attribution International License (CC BY 4.0).

http://creativecommons.org/licenses/by/4.0/

\begin{abstract}
In the following black hole model, electrons and positrons form a neutral gas which is confined by gravitation. The smaller masses are supported against gravity by electron degeneracy pressure. Larger masses are supported by ideal gas and radiation pressure. In each case, the gas is a polytrope which satisfies the Lane-Emden equation. Solutions are found that yield the physical properties of black holes, for the range 1000 to 100 billion solar masses.
\end{abstract}

\section{Keywords}

Black Hole Model, Lepton Gas, Polytrope, Lane-Emden Equation

\section{Introduction}

The discovery of very large black holes in the early Universe is physical evidence that these black holes formed during the Big Bang. The electron-positron model requires vast numbers of positrons, and these were available only during the lepton epoch of the Big Bang [1]. Gravitational collapse and fragmentation must have occurred at that time and later, as the expansion of the Universe continued.

It will be shown in the following that the smaller black holes achieve equilibrium as a degenerate quantum gas. The gas is in its ground state and does not radiate. The radius of such objects decreases with increasing mass, but it cannot be smaller than the Schwarzschild radius, $R_{s}=2 G M / c^{2}$. The gas remains nonrelativistic until that point. Larger masses reach equilibrium as a mixture of ideal gas and radiation. Their radius is equal to $R_{s}$, so that the radiation is confined. The average density decreases as $1 / M^{2}$, and as a result, the gas remains nonrelativistic throughout.

\section{Intermediate-Mass Black Holes}

The Fermi energy for a completely degenerate $(T=0)$ gas of $N / 2$ electrons is given by [2] [3] 


$$
\epsilon_{F}=\left(\frac{3 \pi^{2}}{2}\right)^{2 / 3} \frac{\hbar^{2}}{2 m^{5 / 3}} \rho^{2 / 3}
$$

where $\rho$ is the mass density of the neutral lepton gas. The pressure of the degenerate gas is

$$
P=\left(\frac{3 \pi^{2}}{2}\right)^{2 / 3} \frac{\hbar^{2}}{5 m^{8 / 3}} \rho^{5 / 3}=K \rho^{1+\frac{1}{n}}
$$

Therefore, the gas is a polytrope [4] [5] with $n=1.5$ and

$$
K=\left(\frac{3 \pi^{2}}{2}\right)^{2 / 3} \frac{\hbar^{2}}{5 m^{8 / 3}}=1.71 \times 10^{18} \text { (cgs units) }
$$

A spherically symmetric mass, in hydrostatic equilibrium, satisfies the gravitational field equation

$$
\frac{1}{r^{2}} \frac{\mathrm{d}}{\mathrm{d} r}\left(r^{2} \frac{\mathrm{d} \psi}{\mathrm{d} r}\right)=4 \pi G \rho
$$

as well as

$$
\frac{\mathrm{d} P}{\mathrm{~d} r}=-\rho \frac{\mathrm{d} \psi}{\mathrm{d} r}
$$

Together, they yield the pressure formula

$$
\frac{1}{r^{2}} \frac{\mathrm{d}}{\mathrm{d} r}\left(\frac{r^{2}}{\rho} \frac{\mathrm{d} P}{\mathrm{~d} r}\right)=-4 \pi G \rho
$$

If the mass is a polytropic gas, then

$$
(n+1) K \frac{1}{r^{2}} \frac{\mathrm{d}}{\mathrm{d} r}\left(r^{2} \frac{\mathrm{d} \rho^{1 / n}}{\mathrm{~d} r}\right)=-4 \pi G \rho
$$

Define $\rho=\rho_{0} \theta^{n}$ and substitute to find

$$
\left[\frac{(n+1) K}{4 \pi G \rho_{0}^{1-\frac{1}{n}}}\right] \frac{1}{r^{2}} \frac{\mathrm{d}}{\mathrm{d} r}\left(r^{2} \frac{\mathrm{d} \theta}{\mathrm{d} r}\right)=-\theta^{n}
$$

Finally, define $r=\alpha \xi$ where the constant

$$
\alpha=\left[\frac{(n+1) K}{4 \pi G \rho_{0}^{1-\frac{1}{n}}}\right]^{1 / 2}
$$

in order to obtain the Lane-Emden equation [4] [5]

$$
\frac{1}{\xi^{2}} \frac{\mathrm{d}}{\mathrm{d} \xi}\left(\xi^{2} \frac{\mathrm{d} \theta}{\mathrm{d} \xi}\right)=-\theta^{n}
$$

The function $\theta(\xi)$ and the variable $\xi$ are dimensionless. The initial conditions at the center $(\xi=0)$ are

$$
\theta(0)=1 \text { and }\left.\frac{\mathrm{d} \theta}{\mathrm{d} \xi}\right|_{0}=0
$$


so that $\rho_{0}$ represents the density at the center.

The Lane-Emden equation has been solved numerically for many values of the polytropic index $n$. The following is a summary for the case $n=1.5$. The solution $\theta(\xi)$ decreases monotonically from $\theta(0)=1$ to $\theta\left(\xi_{1}\right)=0$, where $\xi_{1}=3.654$ (from the tables). This corresponds to zero density and pressure at the surface, $R=\alpha \xi_{1}$. This would yield the radius, once the value of $\alpha$ is known. However, a more direct approach is provided by the mass-radius relation [4]

$$
K=N_{n} G M^{(n-1) / n} R^{(3-n) / n}=N_{1.5} G M^{1 / 3} R
$$

The tabulated coefficient $N_{1.5}=0.424$, so that

$$
R=\frac{K}{0.424 G} M^{-1 / 3}=6.05 \times 10^{25} M^{-1 / 3}(\mathrm{~cm})
$$

This shows that the radius decreases with increasing mass. It will continue to do so until the Schwarzschild radius, $R_{s}=2 G M / c^{2}$, is reached at $M=8 \times 10^{6} M_{\odot}$. This defines the largest intermediate-mass black hole.

The average density $\bar{\rho}=3 M / 4 \pi R^{3}$ and the central density $\rho_{0}=5.99 \bar{\rho}$ (tables) are found by substituting (13)

$$
\rho_{0}=6 . \times 10^{-78} M^{2}\left(\mathrm{~g} \cdot \mathrm{cm}^{-3}\right)
$$

while the central pressure is

$$
P_{0}=K \rho_{0}^{5 / 3}=3.9 \times 10^{-112} M^{10 / 3}\left(\mathrm{dyn} \cdot \mathrm{cm}^{-2}\right)
$$

Finally, the Fermi energy at the center is (1)

$$
\epsilon_{F 0}=1.35 \times 10^{-60} M^{4 / 3}(\mathrm{erg})
$$

These physical properties are tabulated below (Table 1).

The central density, pressure, and kinetic energy all increase rapidly with mass. In the final line, $R=R_{s}$.

\section{Supermassive Black Holes}

Black holes of mass greater than $M=8 \times 10^{6} M_{\odot}$ are supported against gravity by ideal gas and radiation pressure. In all cases, $R=R_{s}$ so that the lepton gas and radiation are confined. The pressure is given by

Table 1. Intermediate-mass.

\begin{tabular}{ccccc}
\hline$M$ & $R>R_{s}$ & $\rho_{0}$ & $P_{0}$ & $\epsilon_{F 0}$ \\
\hline$\left(M_{\odot}\right)$ & $(\mathrm{cm})$ & $\left(\mathrm{g} \cdot \mathrm{cm}^{-3}\right)$ & $(\mathrm{Pa})$ & $(\mathrm{eV})$ \\
\hline $10^{3}$ & $4.8\left(10^{13}\right.$ & $2.6\left(10^{-5}\right)$ & $3.9\left(10^{9}\right)$ & 2.1 \\
$10^{4}$ & $2.25\left(10^{13}\right.$ & $2.6\left(10^{-3}\right)$ & $8.4\left(10^{12}\right)$ & $4.5(10)$ \\
$10^{5}$ & $1.05\left(10^{13}\right)$ & $2.6\left(10^{-1}\right)$ & $1.8\left(10^{16}\right)$ & $9.6\left(10^{2}\right)$ \\
$10^{6}$ & $4.8\left(10^{12}\right)$ & $2.6(10)$ & $3.9\left(10^{19}\right)$ & $2.1\left(10^{4}\right)$ \\
$8\left(10^{6}\right)$ & $2.4\left(10^{12}\right)$ & $1.7\left(10^{3}\right)$ & $3.9\left(10^{22}\right)$ & $3.3\left(10^{5}\right)$ \\
\hline
\end{tabular}




$$
P=P_{\mathrm{gas}}+P_{\mathrm{rad}}=\frac{\rho}{m} k T+\frac{a}{3}(k T)^{4}
$$

where $a=\pi^{2} / 15(\hbar c)^{3}$. The mixture may be treated analytically by adopting the following device from the standard stellar model [4]. Define $\beta=P_{\text {gas }} / P$, so that

$$
\beta P=\frac{\rho}{m} k T \text { and }(1-\beta) P=\frac{a}{3}(k T)^{4}
$$

Eliminate $k T$ from these equations to find

$$
P=\left[\frac{3}{a} \frac{1-\beta}{m^{4} \beta^{4}}\right]^{1 / 3} \rho^{4 / 3}=K \rho^{4 / 3}
$$

If $\beta$ is assumed to be a constant, then the mixture is a polytrope of index $n=3$, which satisfies the Lane-Emden Equation (10). The solution for $n=3$ yields the mass-radius relation (12)

$$
K=0.364 G M^{2 / 3}=2.43 \times 10^{-8} M^{2 / 3}(\text { cgs units })
$$

Therefore, the value of $\beta$ is determined by the mass alone

$$
\frac{1-\beta}{\beta^{4}}=\frac{a}{3} K^{3} m^{4}=6.9 \times 10^{-83} M^{2}
$$

The average density of the supermassive black hole $\bar{\rho}=3 M / 4 \pi R_{s}^{3}$ yields the central density (tables)

$$
\rho_{0}=6.93 \bar{\rho}=5.1 \times 10^{83} M^{-2}\left(\mathrm{~g} \cdot \mathrm{cm}^{-3}\right)
$$

The central pressure is

$$
P_{0}=K \rho_{0}^{4 / 3}=1.0 \times 10^{104} M^{-2}\left(\mathrm{dyn} \cdot \mathrm{cm}^{-2}\right)
$$

Finally, the temperature is found from (18) $k T=\beta m K \rho^{1 / 3}$. At the center,

$$
k T_{0}=1.8 \times 10^{-7} \beta(\mathrm{erg})
$$

Physical properties are tabulated below (Table 2).

The central density, pressure, and temperature all decrease with increasing mass. The values of $\beta$ show that radiation pressure dominates in the larger masses.

Table 2. Supermassive.

\begin{tabular}{cccccc}
\hline$M$ & $R=R_{s}$ & $\rho_{0}$ & $P_{0}$ & $k T_{0}$ & $\beta$ \\
\hline$\left(M_{\odot}\right)$ & $(\mathrm{cm})$ & $\left(\mathrm{g} \cdot \mathrm{cm}^{-3}\right)$ & $(\mathrm{Pa})$ & $(\mathrm{eV})$ & \\
\hline $8\left(10^{6}\right)$ & $2.4\left(10^{12}\right)$ & $2\left(10^{3}\right)$ & $3.9\left(10^{22}\right)$ & $1.1\left(10^{5}\right)$ & 0.99 \\
$10^{7}$ & $3\left(10^{12}\right)$ & $1.3\left(10^{3}\right)$ & $2.5\left(10^{22}\right)$ & $1.1\left(10^{5}\right)$ & 0.98 \\
$10^{8}$ & $3\left(10^{13}\right)$ & $1.3(10)$ & $2.5\left(10^{20}\right)$ & $6.2\left(10^{4}\right)$ & 0.62 \\
$10^{9}$ & $3\left(10^{14}\right)$ & $1.3\left(10^{-1}\right)$ & $2.5\left(10^{18}\right)$ & $2.3\left(10^{4}\right)$ & 0.23 \\
$10^{10}$ & $3\left(10^{15}\right)$ & $1.3\left(10^{-3}\right)$ & $2.5\left(10^{16}\right)$ & $8\left(10^{3}\right)$ & 0.08 \\
$10^{11}$ & $3\left(10^{16}\right)$ & $1.3\left(10^{-5}\right)$ & $2.5\left(10^{14}\right)$ & $2\left(10^{3}\right)$ & 0.02 \\
\hline
\end{tabular}




\section{Remarks}

The model presented here is a great improvement over the previous work [1]. The Lane-Emden solutions yield functions for the density, pressure, and kinetic energy. They decrease from their maximum value at the center to zero at the surface of the black hole. The polytropic solution is simply more realistic than the incompressible model.

It is evident from Table 1 and Table 2 that the largest and smallest black holes are similar, in that their density is very low. Moreover, the force of gravity at the surface is weak. It is roughly equal to the force here on Earth. Leptons near the surface would be vulnerable to ambient radiation, and it is an open question whether such black holes could achieve equilibrium in the first place. Such considerations provide loose upper and lower limits for the black hole mass. To date, the largest and smallest observed masses are $2 \times 10^{10} M_{\odot}$ and $5 \times 10^{4} M_{\odot}$, respectively.

Supermassive black holes must be stable, in order to endure for more than 10 billion years. The lepton model is stable, in part, because there are no thermonuclear reactions. For this to remain the case, it is important that the black hole stays free of contamination by baryons. In particular, there can be no flow of matter into the black hole. A recent observation of the Milky Way's black hole shows that this is, indeed, the case [6]. It was found that cool inflowing gas becomes hot and then expands outwardly, before it reaches the black hole. Moreover, the opposing flows are equal in magnitude. These experiments demonstrate that the function of a black hole is to heat hydrogen gas. The black hole steadily loses energy in this process. The most spectacular are the quasars, which can radiate one solar mass per year. In a related development, star formation has been detected in the hot gas outflow from an active galaxy [7].

\section{Conflicts of Interest}

The author declares no conflicts of interest regarding the publication of this paper.

\section{References}

[1] Dalton, K. (2014) The Galactic Black Hole. Hadronic Journal, 37, 241-245. http://vixra.org/abs/1404.0067

[2] Landau, L. and Lifschitz, E. (1980) Statistical Physics Part 1. 3rd Edition, Pergamon Press, Oxford, Sect. 57.

[3] Pathria, R. and Beale, P. (2011) Statistical Mechanics. 3rd Edition, Elsevier, Amsterdam, Chap. 8.

[4] Chandrasekhar, S. (1958) An Introduction to the Study of Stellar Structure. Dover, Mineola, Chap. IV.

[5] Horedt, G. (2004) Polytropes. Kluwer, ¡Alphen aan den Rijn, Sect. 2.1.

[6] Wang, Q., et al. (2013) Dissecting X-Ray-Emitting Gas around the Center of Our Galaxy. Science, 341, 981-983. https://doi.org/10.1126/science.1240755

[7] Maiolino, R., et al. (2017) Star Formation in a Galactic Outflow. Nature, 544, 204-206. https://doi.org/10.1038/nature21677 